\title{
К ВОПРОСУ АДСОРБЦИИ В ГАЗОХРОМАТОГРАФИЧЕСКОЙ КОЛОНКЕ
}

L. MOLDER, O. METLITSKAJA. ADSORPTSIOONIST GAASIKROMATOGRAAFIAKOLONNIS

L. MOLDER, O. METLITSKAYA. ABOUT ADSORPTION IN THE GAS CHROMATOGRAPHIC COLUMN

(Представил О. Эйзен)

При определении термодинамических функций растворов по данным ГЖХ эксперименты обычно проводят, предполагая, что роль адсорбционных процессов в газохроматографической колонке ничтожна и в расчетах ими можно пренебречь. Однако в $\left[{ }^{1,2}\right]$ показано, что при использовании сильно полярных стационарных фаз измеренные значения удельных удерживаемых объемов $\left(V_{g}\right)$ в значительной степени зависят от соотношения неподвижной жидкой фазы и твердого носителя $\left(m_{L} / m_{S}\right)$, причем уменьшение $m_{L} / m_{S}$ приводит к более прочному удерживанию растворенного вещества в колонке. Это явление объяснено процессом адсорбции растворенного вещества на поверхности неподвижной жидкой фазы, и показано, что в данном случае объем удерживания, отнесенный к 1 г насадки $\left(V_{R g}^{0}\right)$, выражается уравнением, состоящим из двух членов, которые характеризуют вклады, определяемые растворимостью и адсорбцией на граничной поверхности жидкости:

$$
V_{R g}^{0}=K_{S} V_{L}+K_{A} A_{L}
$$

где $K_{\mathrm{S}}-$ коэффициент распределения, $K_{A}-$ коэффициент адсорбции, $V_{L}$ и $A_{L}$ - объем и поверхность жидкой фазы, отнесенные к 1 2 насадки, соответственно. $A_{L}$ не только зависит от удельной поверхности твердого носителя, но и является нелинейной функцией соотношения масс жидкой фазы и твердого носителя (с увеличением $m_{L} / m_{S}$ поверхность раздела жидкость-газ уменьшается). Для определения вклада адсорбции необходимо независимым способом установить связь между $A_{L}$ и $V_{L}$, что представляет собой крайне сложную задачу. Кроме того, справедливость объяснения непостоянства $V_{g}$ (нелинейной зависимости $V_{R g}^{0}$ от $V_{L}$ ) явлением адсорбции только на поверхности неподвижной жидкой фазы весьма относительна.

В настоящей работе предпринята попытка объяснить нелинейную зависимость $V_{R g}^{0}$ от количества жидкой фазы в колонке также другими явлениями, в том числе адсорбцией на твердом носителе и неравномерностью слоя неподвижной жидкой фазы на носителе. Область линейной зависимости (рисунок, $a) V_{R g}^{0}$ от $V_{L}$ соответствует условиям, когда вклад адсорбции ничтожен. Изменение $A_{L}$ с изменением $V_{L}$.практически не влияет на $V_{R g}^{0}$. Нелинейная зависимость при малых значениях $V_{L}$ может объясняться только неравномерным покрытием жидкостью поверхности носителя. В результате этого 


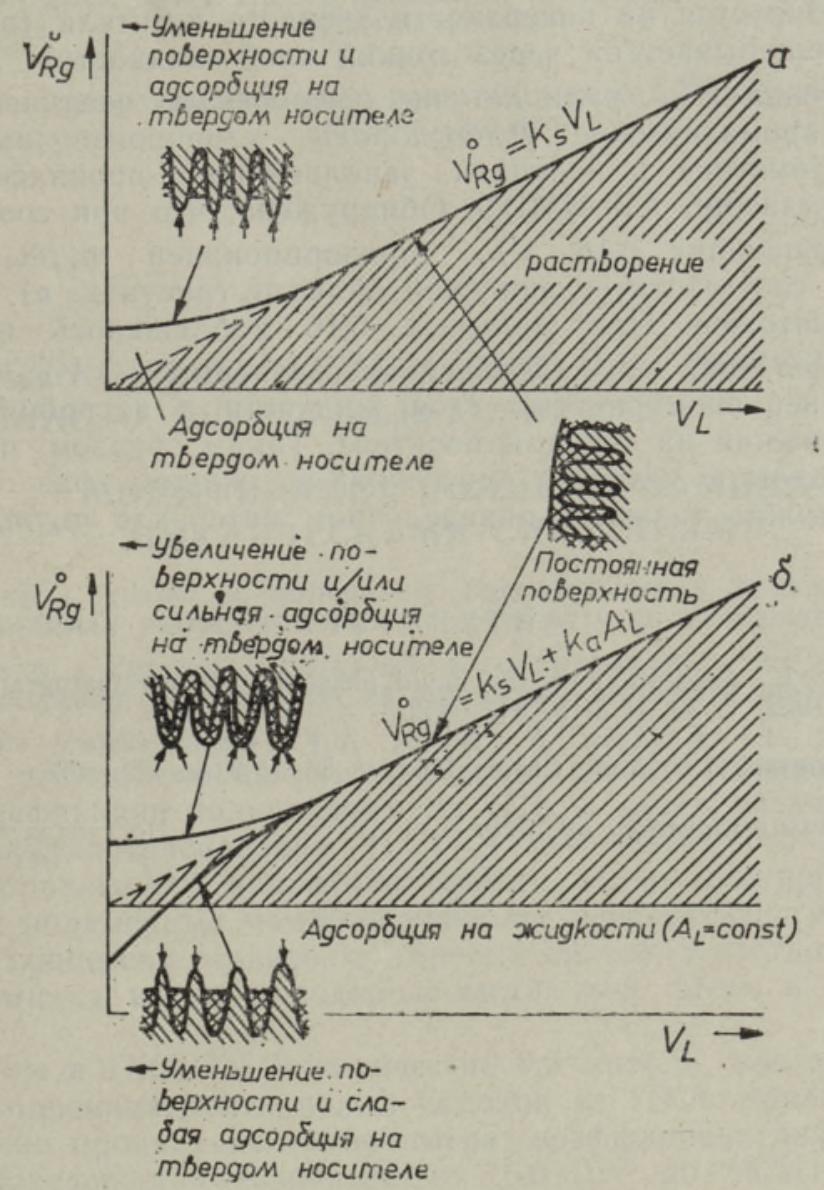

Связь между $V_{R g}^{0}$ и $V_{L}$ в зависимости от характера адсорбции в газохроматографической колонке.

растворенное вещество адсорбируется на участках поверхности носителя, не покрытых слоем жидкости достаточной толщины. Это можег иметь место при низкой адсорбционной способности носителя по отношению к неподвижной жидкой фазе, особенно если насадка изготовляется непосредственно внутри колонки.

Область линейной зависимости (рисунок, б) $V_{R g}^{0}$ от $V_{L}$ соответствует условиям, когда $A_{L}$ не зависит от $V_{L}$ (все мелкие поры заполнены жидкостью). Отрезок, отсекаемый прямой на оси $V_{R g}^{0}$, соответствует вкладу адсорбции в $V_{R g}^{0}\left(K_{A} A_{L}\right)$. Вклад растворения $\left(K_{S} V_{L}\right)$ характеризуется высотой заштрихованной области. Нелинейная зависимость при малых значениях $V_{L}$ объясняется как изменением поверхности раздела газ-жидкость, так и адсорбцией на твердом носителе. Отрицательное отклонение значений $V_{R g}^{0}$ от линейности наблюдается в том случае, если уменьшение $V_{L}$ приводит к уменьшению $A_{L}$ (носитель неравномерно покрыт жидкостью) и коэффициент адсорбции растворенного вещества на освободившейся поверхности носителя меньше, чем на поверхности жидкости, т. е. адсорбция на жидкости не компенсируется адсорбцией на твердом носителе.

Положительное отклонение $V_{R g}^{0}$ от линейности имеет место, если уменьшение $V_{L}$ приводит к увеличению $A_{L}$ или растворенное вещество 
сильно адсорбируется на поверхности твердого носителя (поверхность носителя «прощупывается» через тонкий слой жидкости). Нами проведены измерения $V_{R g}^{0}$ ряда летучих органических соединений (алканы, алкены, ароматические углеводороды, хлорпроизводные углеводородов, нитрометан) в колонках, заполненных 4-пропилфенолом на носителе - хезасорб AW-HMDS. Обнаружено, что при соотношениях $m_{L} / m_{S}$, превышающих $0,16, V_{R g}^{0}$ пропорционален $m_{L} / m_{S}$ (постоянный $V_{g}$ ), что соответствует линейной области (рисунок, $a$ ), где вклад адсорбции ничтожен. При $m_{L} / m_{S}<0,16$ наблюдалось постепенноє увеличение значений $V_{g}$ (нелинейная зависимость $V_{R g}^{0}$ ), которое объясняется неравномерностью слоя жидкости и адсорбцией растворенного соединения на твердом носителе. Таким образом, при использовании фенолов в качестве неподвижных жидких фаз пренебречь адсорбцией можно только в определенном интервале $m_{L} / m_{S}$.

\section{ЛИТЕРАТУРА}

1. Martin, R. L. Adsorption on the liquid phase in gas chromatography. - Anal. Chem., 1962, v. $33, \mathrm{~N} 3$, p. $347-352$.

2. Pecsok, R. L., Yllana, A., Aziz, A.-K. Liquid surface effects in gas chromatography. - Anal. Chem., 1964, v. 36, N 3, p. $452-457$.

талличский политехнический институт

Поступила в редакцию 1/IV 1982 